\title{
POSTe-IP: Aplicativo Web para Otimização do Sistema e das Tecnologias de Iluminação Pública
}

\author{
Lucas Mudo de Araujo ${ }^{1}$, Pedro Henrique Campagna Moura da Silva ${ }^{1}$, \\ Luiz Fernando Delboni Lomba ${ }^{1}$, Wesley Eiji Sanches Kanashiro ${ }^{1}$ \\ ${ }^{1}$ Instituto Federal de Mato Grosso do Sul - IFMS \\ Campus Campo Grande \\ CEP: 79.100-510 - Campo Grande - Mato Grosso do Sul - Brasil \\ \{lucasaraujoms1507, pedrohomds\}@gmail.com, \\ \{luiz.lomba, wesley.kanashiro\}@ifms.edu.br
}

\begin{abstract}
The purpose of this research is to develop a Web application that receives data collected from the public lighting system and allows the its management using software. The data will be collected by sensors present in several platforms distributed on the park of light and transmitted to the application central server through a wireless network sensors. The system will be able to to consult the ideal luminous intensity of each pole, besides the actual energy consumption and the status of each one. This paper presents a resume of the proposed system structure.
\end{abstract}

Resumo. O objetivo desta pesquisa é desenvolver uma aplicação Web que receba os dados coletados do sistema de iluminação pública e permita o gerenciamento do sistema via software. Os dados serão coletados por sensores presentes em diversas plataformas distribuídas no parque de iluminação e transmitidos ao servidor central da aplicação, por meio de uma rede de sensores sem fio. $O$ sistema permitirá a consulta da intensidade luminosa ideal de cada poste, além do consumo energético real e o status de cada um. Este artigo apresenta um resumo da estrutura proposta do sistema.

\section{Introdução}

Da busca de soluções inovadoras e sustentáveis para suprir as necessidades advindas da expansão constante da população, em especial nas cidades, surge o conceito de cidades inteligentes (smart cities), promovidas com a utilização das Tecnologias de Informação e Comunicação. A proposta das smart cities é fazer um melhor uso dos recursos públicos, de modo que aumente a qualidade dos serviços oferecidos aos cidadãos, ao mesmo tempo em que reduz os custos operacionais [Zanella et al. 2014].

Dentre os principais componentes de uma cidade, destaca-se o sistema de iluminação pública (SIP). O modelo atual do SIP se mostra simplório, pois avalia somente a iluminação natural, atuando de forma binária: ligado ou desligado. Reduzindo, dessa forma, as possibilidades de economia em situações onde não há necessidade da lâmpada operar com $100 \%$ de sua intensidade. Outra problemática é o cálculo do consumo energético, realizado pelas concessionárias de energia considerando um consumo médio diário de 11 horas e 52 minutos [ANEEL 2010]: este modelo considera que todas 
as lâmpadas ficam acesas durante o período (sem excluir aquelas que apresentam problemas) e possuem o mesmo consumo energético. Surge como uma forma de solucionar tal problemática a utilização de técnicas de sensoriamento e tomada de decisão inteligente.

A modernização dos parques de iluminação permite maior eficiência energética e qualidade de serviço, além de diminuir os custos (expensa de energia) e criar importantes indicadores a partir dos dados coletados [da Silva 2006]. Neste contexto, este trabalho tem como objetivo desenvolver uma aplicação Web que receba os dados coletados do SIP, calcule a intensidade luminosa ideal para cada poste e apresente o consumo energético deles.

\section{Trabalhos Relacionados}

Um sistema que utiliza sensores infravermelhos para detectar o fluxo nas vias, para então ativar a iluminação, foi proposto [Jagadeesh et al. 2015] intentando reduzir o consumo de energia em momentos em que não há fluxo nas ruas. Outro sistema foi apresentado [Müllner and Riener 2011] com o objetivo de controlar a iluminação pública baseado na presença das pessoas, mas com a inovação de utilizar o smartphone do pedestre como sensor para indicar sua presença na região que a iluminação deve ser ativada, descartando o uso de sensores adicionais. Por fim, outro sistema [Leccese et al. 2014] baseia o controle da iluminação pública na ausência de luz natural e presença de carros e/ou pedestres na área de interesse, utilizando redes ZigBee e WiMAX.

Os trabalhos apontam que aplicações deste tipo têm grande potencial para revolucionar o sistema de iluminação pública, permitindo realizar o gerenciamento do mesmo de forma eficiente e contribuindo para a economia de energia.

\section{O Sistema POSTe-IP}

O sistema é chamado de POSTe-IP (Projeto de Otimização do Sistema e das Tecnologias da Iluminação Pública) e tem como objetivo auxiliar no gerenciamento dos parques de iluminação, de forma a obter a maior eficiência energética. Para tal, o mesmo analisa dados coletados por plataformas espalhadas pelo SIP, calcula a quantidade luminosa necessária para determinada região e o consumo energético real de cada poste. A arquitetura proposta é apresentada na Figura 1.

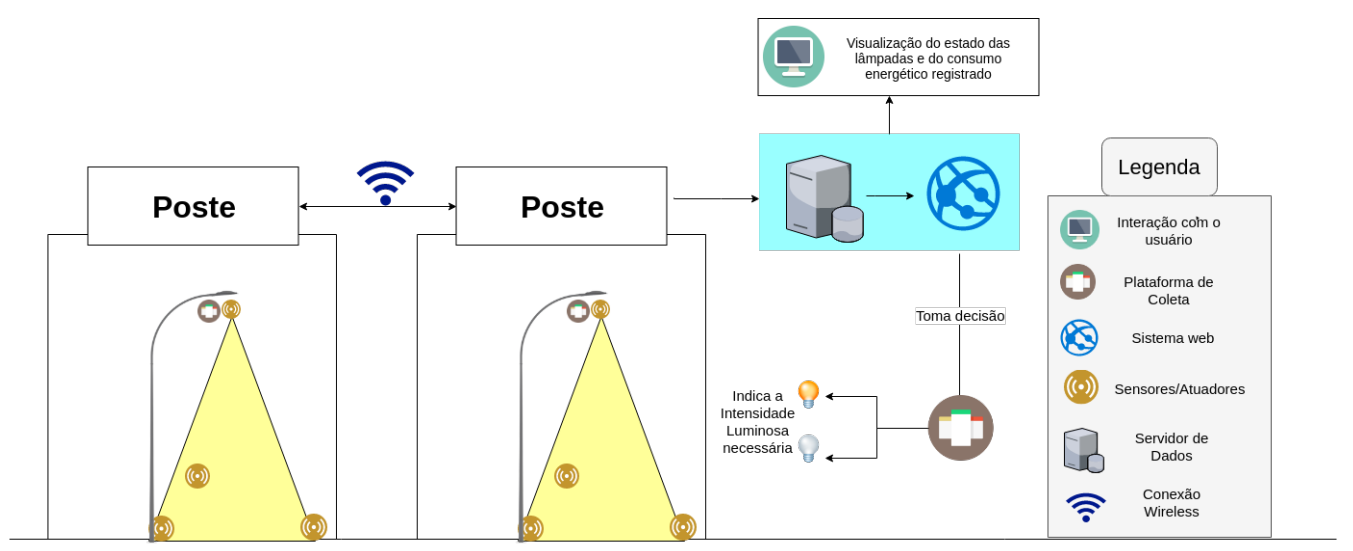

Figura 1. Arquitetura proposta para o sistema POSTe-IP. 
O cenário é formado por estações de coleta de dados, distribuídas por um determinado parque de iluminação pública, compostas por um microcontrolador (Arduino) e sensores, que coletam a luminosidade (TSL2541), corrente elétrica (AC712-30A), tensão elétrica (P8) e presença de pessoas (RCWL-0516). Os dados coletados são transmitidos por uma rede de sensores sem fio (formada por módulos XBee) até chegarem ao servidor central que roda a aplicação.

Para a tomada de decisão, ou seja, indicar quando uma lâmpada deve ser ativada e em qual intensidade, será utilizada a norma brasileira de iluminação técnica (ABNT NBR 5101), que estabelece os requisitos mínimos para iluminação das vias públicas. A Figura 2 apresenta o fluxo para tomada de decisão: após iniciar o sistema, verifica-se a intensidade luminosa e a compara com o padrão estabelecido na norma técnica; se a intensidade estiver dentro do mínimo, verifica-se o fluxo de veículos/pedestres no local, caso contrário ajusta-se a intensidade da lâmpada para o valor mínimo estabelecido; se há fluxo no local, verifica-se novamente a intensidade luminosa (ela deve estar dentro do adequado para locais com fluxo); se a intensidade não estiver adequada, ela é ajustada; caso contrário verificasse a existência de fluxo; quando não houver mais fluxo o sistema retorna ao estado inicial, ajustando a intensidade luminosa para a faixa mínima.

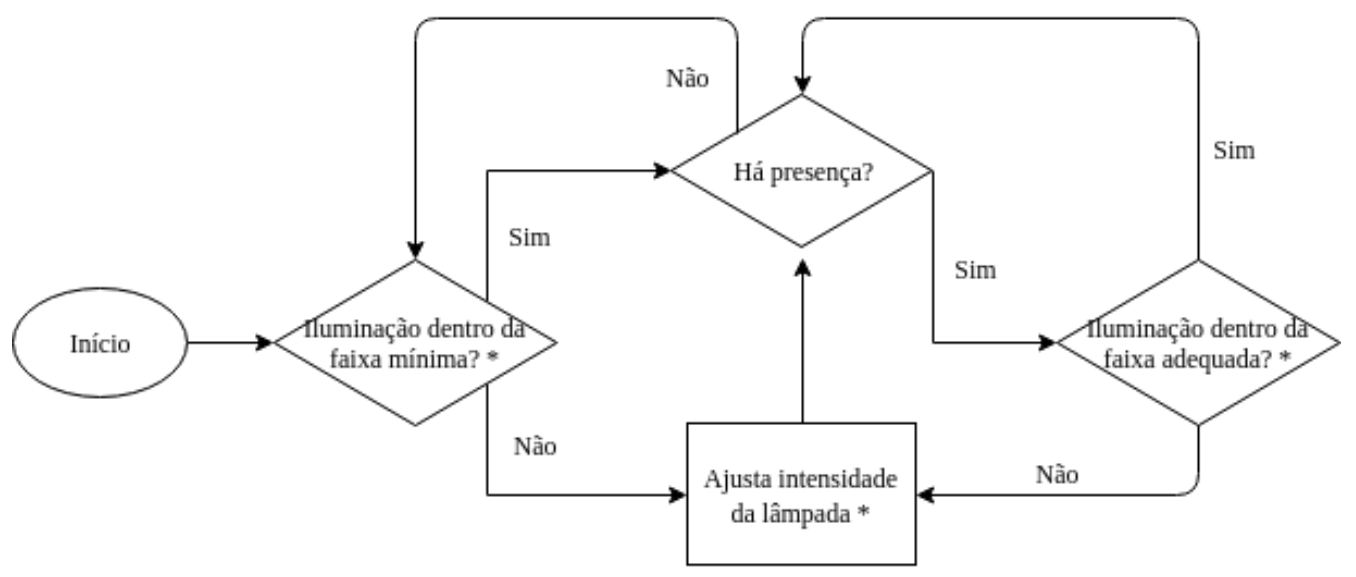

*De acordo com os valores estipulados pela norma ABNT NBR 5101

Figura 2. Fluxo para tomada de decisão do sistema.

Dentre as funcionalidades do sistema destacamos o dashboard. Ele é o painel de controle onde estarão disponíveis as informações sobre: postes acesos (ou com problema) em determinado momento; consumo de energia de cada poste; variação da intensidade de um poste num certo período; e comparativo entre a intensidade luminosa atual e ideal.

Para o desenvolvimento estão sendo utilizadas a linguagem de programação PHP para o back-end, HTML e CSS para ofront-end e o banco de dados MySQL. O processo de desenvolvimento utilizado é o incremental, sendo que no $1^{\circ}$ incremento foram implementadas as telas de apresentação e de cadastros. A Figura 3 mostra a tela de visão geral do POSTe-IP e uma das telas de cadastro implementadas. Atualmente está em desenvolvimento o $2^{\circ}$ incremento, que contempla a hospedagem do Web site e a implementação das funções contempladas no dashboard. 


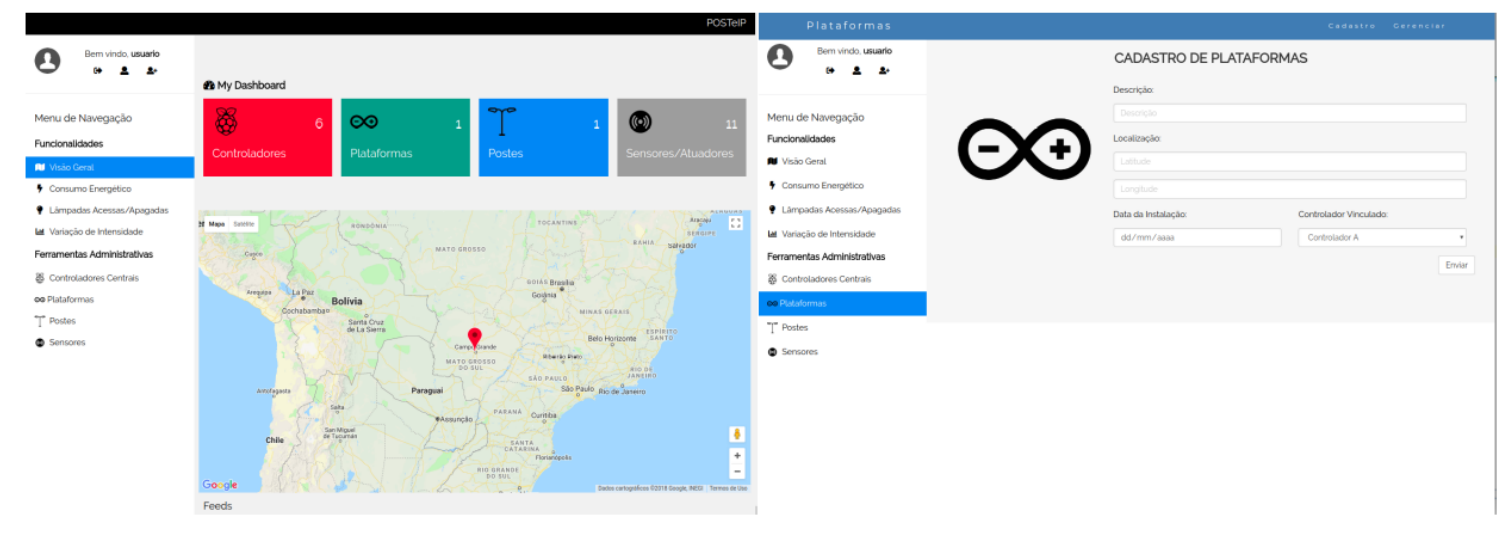

Figura 3. Telas do sistema.

\section{Considerações Finais}

Os resultados das prospecções realizadas mostram a necessidade de mudanças no sistema de iluminação pública atual, em especial quanto ao gerenciamento do consumo energético. Foram encontrados trabalhos similares, que buscaram alternativas ao modelo atual, motivando o desenvolvimento deste trabalho.

O trabalho está em andamento, atualmente no desenvolvimento das funcionalidades do $2^{\circ}$ incremento. Os próximos passos envolvem a implementação das funcionalidades do dashboard e a integração com as plataformas de coleta de dados (não apresentadas neste trabalho).

\section{Referências}

ANEEL (2010). Resolução normativa no 414, de 09 de setembro de 2010, que estabelece as condições gerais de fornecimento de energia elétrica de forma atualizada e consolidada.

da Silva, L. L. F. (2006). Iluminação Pública no Brasil: Aspectos energéticos e institucionais. PhD thesis, UFRJ, Rio de Janeiro.

Jagadeesh, Y., Akilesh, S., Karthik, S., and Prasanth (2015). Intelligent street lights. Procedia Technology, 21:547 - 551. Smart Grid Technologies.

Leccese, F., Cagnetti, M., and Trinca, D. (2014). A smart city application: A fully controlled street lighting isle based on raspberry-pi card, a zigbee sensor network and wimax. Sensors, 14(12):24408-24424.

Müllner, R. and Riener, A. (2011). An energy efficient pedestrian aware smart street lighting system. International Journal of Pervasive Computing and Communications, 7(2):147-161.

Zanella, A., Bui, N., Castellani, A., Vangelista, L., and Zorzi, M. (2014). Internet of things for smart cities. IEEE Internet of Things Journal, 1(1):22-32. 